\title{
Sensitivity of the gamma band auditory steady state response to linguistic features of a stimulus
}

\author{
Jessamy Norton-Ford*, Siyi Deng ${ }^{\dagger}$, Mary Louise Kean ${ }^{\dagger}$ and Ramesh Srinivasan ${ }^{\dagger}$
}

\section{Introduction}

An auditory steady state response (aSSR) is a measurable output, either electromagnetically or magnetoencephalographically, of neural populations oscillating synchronously with an incoming auditory stimulus. Classically, auditory steady state responses have been induced by click-trains (Galambos et al., 1974) or amplitude/frequency modulated tones (Picton et al., 1987). The presence or absence of a steady state response is determined by the power of the measured response at a frequency of interest, determined by a fast fourier transform. Critically, a steady state response is represented by a significant amount of power at the modulation frequency of a stimulus. Specific modulation frequencies are chosen based on the unique cognitive processes thought to correspond with a particular frequency band, as well as the individual frequency(ies) within that band which have been shown to elicit the greatest power response. This work deals exclusively with gamma band frequencies $(30 \geq \mathrm{Hz}$ ), with a specific modulation frequency of $40 \mathrm{~Hz}$ (Rickards \& Clark, 1984). This choice of frequency band was based on previous work showing phase-reset sensitivity of naturally occurring gamma band activity to sensory stimuli (Galambos et al., 1981).

A recent study of the gamma band steady state response ( $\gamma$-aSSR) has found significant hemispheric differences in power output based on what is characterized as the "meaningfulness" of the stimulus (Deng \& Srinivasan, 2010). This conclusion has been drawn from findings of a reduction in left hemispheric response (from temporal-parietal areas) when participants listen to speech or speech-with-noise, compared with reversed speech, white noise, or $1000 \mathrm{~Hz}$ tones (all stimuli amplitude-modulated at $40 \mathrm{~Hz}$ ). While a characterization of the difference between speech and reversed speech stimuli does certainly include a presence or lack of conceptual meaning (respectively), there exist other, potentially significant differences between these stimuli which may be contributing to the lateralization effect observed in Deng \& Srinivasan (2010). In addition to the meaning differences present in the comparison of speech and reversed speech conditions, much of the structural information (syntactic or prosodic) that a listener uses to determine that they are listening to a speech signal is highly distorted or absent in the reversed speech case. Of additional interest are studies which have shown a significant left hemisphere reduction of $20 \mathrm{~Hz}$ auditory steady state response (and, subsequent harmonics of this response), based solely on the level of attentiveness of the participant (Müller et al., 2009). Therefore, to confirm that hemispheric asymmetries of the gamma band steady state response $(\gamma$-aSSR $)$ arise as a result of an asynchronous processing of conceptual meaning, and not as either a result of the processing of syntactic/phonetic information or of other experimental variables, this work compares responses to fine-grained linguistic distinctions in a target-monitoring paradigm.

\footnotetext{
${ }^{*}$ Department of Cognitive Science; 3151 Social Science Plaza A; University of California, Irvine; Irvine, CA 92697-5100; jnortonf@uci.edu

${ }^{\dagger}$ Department of Cognitive Science, University of California, Irvine
} 


\section{Methods}

\subsection{Participants}

This study had a total of six participants, three men and three women, average age 33 years, all right-handed native English speakers.

\subsection{Design}

Experimental stimuli included grammatical English sentences, "Jabberwocky" sentences that contained pseudowords in place of content words, reversed speech, and English sentences made "unintelligible" by local segment reversals in a manner developed in Saberi \& Perrott (1999). As a result, the contrasts illustrated in Table 1 could be made.

Table 1: Experimental Contrasts

\begin{tabular}{|l|c|c|c|}
\hline Stimulus type & Conceptual meaning & Syntactic structure & Phonological structure \\
\hline English & yes & yes & yes \\
Jabberwocky & no & yes & yes \\
Reversed Speech & no & no & yes (formant transitions) \\
Unintelligible & no & no & no \\
\hline
\end{tabular}

\section{Task}

During each trial, participants were asked to monitor a $\sim 50$ second stimulus block for a unique target word, which appeared between 1 and 6 times during that block. Following each trial, participants reported the number of times a target was identified by selecting a numeric response on a keyboard placed at a table in front of them.

\section{Regions of Interest}

Symmetric groups of 12 electrodes from each hemisphere were selected for statistical analysis, based on the intersection of the most powerful electrode responses across participants and predetermined regions of interest in temporal and parietal areas.

\subsection{Materials \& Apparatus}

\section{English Stimuli}

Twenty-four English sentences were created, composed of eight monosyllabic words each, designed according to the framework: [[the boy/girl $\left.]_{N P}\left[\left[\mathrm{~V}_{\text {trans }} \text { the } \mathrm{N}\right]_{V P}[\mathrm{P} \text { the } \mathrm{N}]_{P P}\right]_{V P}\right]_{I P}$ (ex., The girl dyed the shirt in the tub). All nouns and verbs were chosen from the middle quartiles of their respective distributions (Vaden et al., 2005).

Jabberwocky Stimuli

Jabberwocky stimuli were created by replacing all nouns and verbs of English stimuli with monosyllabic pseudowords using the ARC Nonword Database (Rastle, 2002), and were "matched" to English sentences by their average categorical densities.

\section{Reversed Speech Stimuli}

Reversed speech stimuli were full-sentence reversals of English stimuli (Matlab 7.2).

\section{Unintelligible Stimuli}

Unintelligible stimuli were created by a concatenation of local (145ms) segment-reversals of English stimuli (Matlab 7.2).

\section{Post-Processing}

During presentation to participants, stimuli were modulated using methods adopted from Deng \& Srinivasan (2010), in which a modulator signal is generated by passing a $40 \mathrm{~Hz}$ 
sinusoid and a $40 \mathrm{~Hz}$ square wave of identical phase and frequency, with a $100 \%$ modulation depth and a $50 \%$ duty-cycle, to a non-linear transfer function.

\section{EEG Recording}

EEG data was collected using a Geodesic Sensor Nets (Electrical Geodesics, Inc.) containing $128 \mathrm{Ag} / \mathrm{AgCl}$ electrodes (each housed in a sponge and mounted on an elastic tension network), and an Advanced Neural Technology amplifier, at a rate of $1024 \mathrm{~Hz}$.

\subsection{Procedure}

During a participant's session there were a total of 36 trials, comprised of nine trials of each of the four stimulus-types (English, Jabberwocky, Reversed speech, Unintelligible). During the stimulus block of each trial, participants heard a random ordering of 21 tokens of six, randomly selected sentences from one of the four possible conditions (English, Jabberwocky, Reversed Speech or Unintelligible), each randomly designated to repeat between 1-6 times without replacement (ex., English, [6 3645545434326655662 1])

\section{Data post-production}

Following data collection, a fast Fourier transform was performed to transform the data into a representation of it's spectral components. Each participant's data was then normalized to their own spontaneous cortical activity by determining a signal-to-noise-ratio of the modulation frequency to the mean of a frequency band $\left(f_{A M} \pm 40 \triangle f\right)$ which excludes the modulation frequency (Deng \& Srinivasan, 2010). Finally, each subject's data was normalized for inter-participant variation in overall power by dividing each participant's steady state response value by the mean steady state response of all participants. Strength of the auditory steady state response was defined as the power (Fourier coefficient) of the modulation frequency component $\left(f_{A M}\right), 40 \mathrm{~Hz}$.

\section{Results}

Figure 1 shows topographical representations of the average signal-to-noise ratio (SNR) power of all participants for each of the four experimental conditions.

ENGLISH

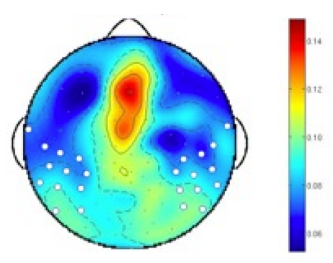

JABBERWOCKY

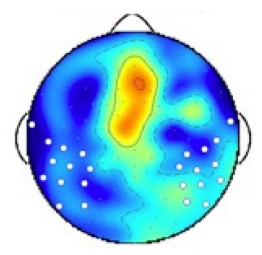

UNINTELLIGIBLE

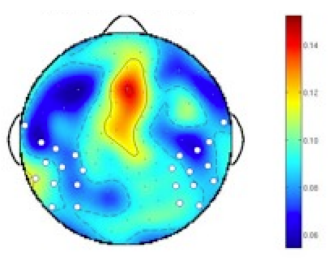

REVERSED

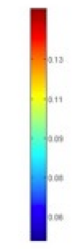

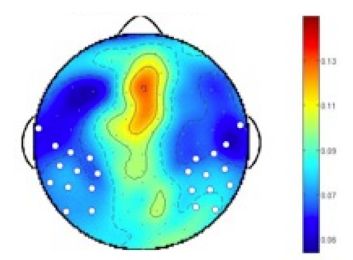

Figure 1: SNR results: Average across all participants by condition

Table 2 shows the results of pair-wise comparisons of the average hemispheric output of each stimulus condition, as well as of the response to the the four stimulus conditions within the left hemisphere alone.

\section{Discussion}

Overall, this work has found that left hemisphere gamma band auditory steady state responses demonstrate sensitivity to linguistic aspects of a stimulus, especially the pres- 
Table 2: Between-Hemisphere and Within (Left) Hemisphere Contrasts

\begin{tabular}{|l||c||c|c|c|c|}
\hline & Left vs. Right & English & Jabberwocky & Reversed & Unintelligible \\
\hline English & $p<0.001^{* * * 1}$ & - & $p=0.0081^{* 2}$ & $p=0.0191$ & $p=0.4281$ \\
\hline Jabberwocky & $p<0.001^{* * *}$ & - & - & $p=0.7013$ & $p=0.0666$ \\
\hline Reversed Speech & $p<0.001^{* * *}$ & - & - & - & $p=0.1369$ \\
\hline Unintelligible & $p<0.001^{* * *}$ & - & - & - & - \\
\hline
\end{tabular}

[1] Bonferroni adjusted $\alpha: p<0.0001^{* * *} \quad$ [2] Bonferroni adjusted $\alpha: p<0.008333^{*}$

ence/absence of conceptual meaning. Within the left-hemisphere, power of ( $\gamma$-aSSR) was significantly greater in response to English vs. Jabberwocky stimuli (a difference in conceptual meaning), and the response to English vs. Reversed speech stimuli condition (which differed in conceptual meaning and syntactic structure) approached significance at the $\alpha=0.05$ level (Bonferroni adjusted; $p=0.0191$ ). In addition, results of this work show an overall right-lateralized response to the four stimulus-types (English, Jabberwocky, Reversed speech and Unintelligible), significant at the $\alpha=0.05$ level (Bonferroni adjusted; $p<0.001$ ). This finding is in-line with previous results of a right-lateralized response to linguistic stimuli, and is not attributable to participant inattentiveness based on performance in the target-monitoring task.

\section{References}

Deng, S., \& Srinivasan, R. (2010). The effect of meaningfulness on gamma band steady-state auditory evoked potential. (working paper) University of California, Irvine.

Galambos, R., Makeig, S., \& Talmachoff, P. J. (1981). A 40-hz auditory potential recorded from the human scalp. Proceedings of the National Academy of Sciences, 78(4), 2643-2647.

Galambos, R., Picton, T. W., Hillyard, S. A., \& i. Krausz, H. (1974). Human auditory evoked potentials i: Evaluation of components 1. Electroencephalography and Clinical Neurophysiology, (pp. 179-190).

Joliot, M., Ribary, U., \& llinas, R. (1994). Human oscillatory brain activity near 40 hz coexists with cognitive temporal binding. Proceedings of the National Academy of Sciences, 91, 11748-11751.

Müller, N., Schlee, W., Hartmann, T., Lorenz, I., \& Weisz, N. (2009). Top-down modulation of the auditory steady-state response in a task-switch paradigm. frontiers in Human Neuroscience.

Picton, T. W., Skinner, C. R., Champagne, S. C., Kellett, A. J. C., \& Maiste, A. C. (1987). Potentials evoked by the sinusoidal modulation of the amplitude or frequency of a tone. Journal of the Acoustic Society of America, 82(1), 165-178.

Rastle, H. J. . C. M., K. (2002). 358, 534 nonwords: The arc nonword database. Quarterly Journal of Experimental Psychology, (55A), 1339-1362.

Rickards, \& Clark (1984). Steady state evoked potentials to amplitude modulated tones., (pp. 163168). Boston: Butterworth Publishers.

Saberi, K., \& Perrott, D. (1999). Cognitive restoration of reversed speech. Nature, 398, 760.

Vaden, K., Hickok, G., \& Halpin, H. (2005). Irvine phonotactic online dictionary.

URL http://lcbr.ss.uci.edu/iphod/index.html 